POP. KULT UR UNDKRITIK

HEFT16 FRÜHLING 2020

\title{
IN H ALT
}

\author{
R U B R I KEN : \\ ZUR ZEIT \\ ESSAYS
}

\section{ZUR ZEIT}

\author{
Miriam Zeh \\ T I K T O K
}

S. 10

Andreas Gebesmair

MAKE OR BUY? BESCHAFFUNGSSTRATEGIEN VON APPLE TV+

S. 16

Wolfgang Ullrich

GRENZÖFFNUNG: NEUE ÜBERGÄNGE ZWISCHEN KUNST UND MODE, , HIGH, ODER, LOW

S. 22 


\title{
Nicolas Pethes
}

\section{T R A I N I E R E N}

S. 30

\section{Daniel Hornuff}

DIE FRAGW ÜRIGKEIT DES GUTEN DESIGNS

$$
\text { S. } 35
$$

\section{Annekathrin Kohout}

\begin{abstract}
KOLUMNISTENALS ONE-MAN-SHOWS
\end{abstract}

$$
\text { S. } 41
$$

\section{Kai-Uwe Hellmann}

WISSENSCHAFTSMARKETING: ANTRAGSPROSA, BEUTEGEM I NSCHAT, PLACEBOEFFKT

$$
\text { S. } 49
$$

\section{Lars Koch / Solvejg Nitzke}

"CHERNOBYL U N D DE HALBWERTSZEITEN DER A N G S T

$$
\text { S. } 55
$$

Gunnar Schmidt

\section{GES I C H T S V ERKE N N U N G}

$$
\text { S. } 61
$$

\section{Paula-Irene Villa Braslavsky}

I D E N T I T Ä T S P O L I T I K

$$
\text { S. } 70
$$

\section{Michelle Janßen}

\section{HASS ALS EVENT: EIN BLICK AUF DIE} G A M I N G - S Z E N E

$$
\text { S. } 77
$$

\section{Lisa Andergassen / Diana Weis}

HORRIBLE MEN - AUSSAGEN UND BERICHTE ÜBER PRINZ ANDREW UND HARVEY WEINSTEIN

$$
\text { S. } 81
$$




\section{Jan Hildebrandt}

H O L L O BOLLO

S. 89

Ramón Reichert

\# FOODPOR AUF INSTAGRAM: WETTBEWERB UND

S OZI ALS T E UER U N G

S. 93

Andreas Rauscher

DIE PHANTOME DES INTERAKTIVEN FILMS S. 100

Maren Lickhardt

DER F IL M IN SERIE

S. 107

Thomas Hecken

P O P U ÄR, P OP, REA A PE P LE

S. 116

\section{ESSAYS}

Thomas Hecken

AUSLAND. ZUR POLITIK DER FREMDE

S. 128

Heinz Drügh

TENDERING TARANTINO: ÄSTHETIK UND KULTURPOETIK (EINE PARTY)

S. 146

HIN WEIS ZU DEN

A U T ORINNEN UNDAUTOREN

S. 177

I M P R E S S U M

S. 179 


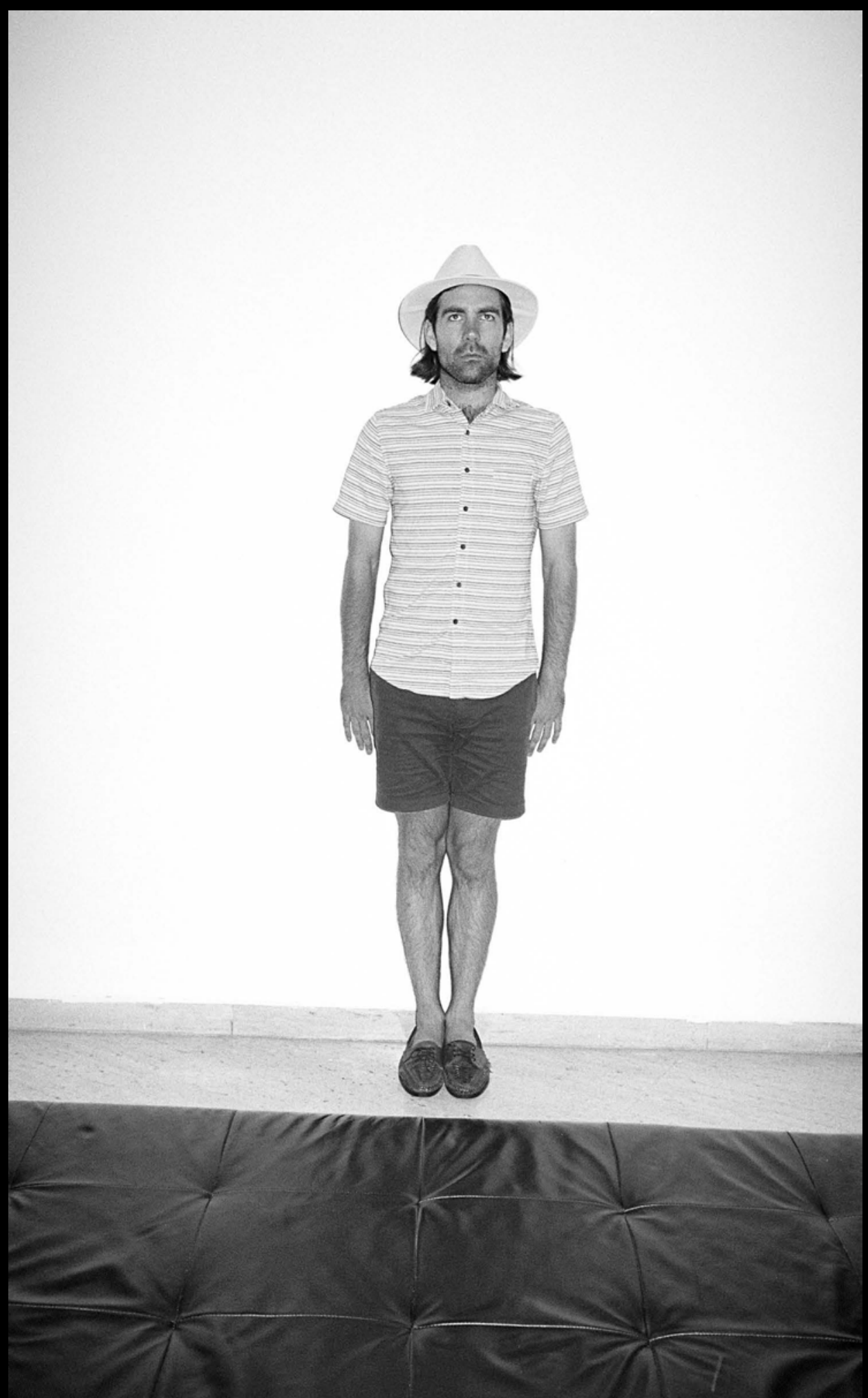

CURATOR MELFIE @ Jackson Eaton 
